\title{
Evaluación de la fenología en vivero de cinco especies arbustivas forrajeras, utilizando productos enraizadores
}

Phenology study in nursery of five forage shrub species, using rooting products

Sánchez Parra William¹, Betancourt Amaya Camilo Ernesto ${ }^{1}$, Lozada Ibarra Claudia del Pilar ${ }^{2}$, Céspedes Sanabria Daniel Alexander ${ }^{2}$ y Roa Vega María Ligia ${ }^{3}$

${ }^{1}$ Licenciados en Producción Agropecuaria, Unillanos, ${ }^{2} \mathrm{MVZ}$, Esp. (c)MSc.

Docentes Unillanos y ${ }^{3}$ Zoot, MSc. Docente Unillanos

\section{clozada@unillanos.edu.co}

Recibido 12 de Diciembre 2014, Aceptado 26 de Septiembre 2015

\section{RESUMEN}

Este trabajo se desarrolló en la Universidad de los Llanos, sede Barcelona km 12 vía Puerto López, donde se sembraron cinco especies en vivero, camarón rojo (Megakespasma erytronchlamys), botón de oro (Tithonia diversifolia), nacedero (Trichanthera gigantea), cayeno (Hibiscus rosa sinensis) y mussaenda rosa (Mussaenda alicia), se utilizaron enraizadores sistémicos, orgánicos y fertilizantes que mejoran la expresión genética, a la vez que aceleran la fenología de la planta, esto con el fin de obtener mejores y mayores producciones. Se utilizó compostaje de 60 días de preparado con un buen porcentaje de biomasa y materia orgánica como sustrato para el llenado de bolsas de polietileno de $25 \times 30 \mathrm{~cm}$ con capacidad para $1 \mathrm{~kg}$. Además se empleó un zarando para minimizar partículas grandes que pudieran interrumpir el desarrollo de la raíz. Se sembraron 350 estacas de $40 \mathrm{~cm}$ distribuidas en un diseño en bloques completamente al azar, se separaron en cinco grupos cada especie con 70 estacas. Los tratamientos fueron T0 (testigo, sin aplicación de enraizador), T1 (vinagre), T2 (hormonagro), T3 (aloe vera) y T4 (roca fosfórica), se realizaron evaluaciones a los 15, 30. 45, 60. 75, 90 y 105 días, de las siguientes variables: altura de la planta $(\mathrm{cm})$, diámetro del tallo de la planta $(\mathrm{cm})$, peso de las raíces $(\mathrm{g})$ y número de las mismas. El crecimiento del botón de oro fue superior $(\mathrm{P}<0.05)$ en todos los tratamientos en comparación con las otras forrajeras, siendo mayor su longitud en el testigo $(65.55 \mathrm{~cm})$, en T0 se 
observa diferencia de más de $20 \mathrm{~cm}$ con relación a los tratamientos que utilizaron los productos. El aumento de longitud a los 15 días de sembrado el botón de oro en TO fue de $15 \mathrm{~cm}$, alcanzando $35 \mathrm{~cm}$ a los 30 e incrementando gradualmente hasta los $65.5 \mathrm{~cm}$ a los 105 días, mientras las otras especies a esta misma edad, medían menos de $30 \mathrm{~cm}$, demostrando un crecimiento similar y lento.

Palabras clave: Leguminosas, forraje, desarrollo radicular.

\section{ABSTRACT}

This work was developed at the University of the Llanos, Barcelona located km 12 road Puerto López, where five species were planted in the nursery, Megakespasma erytronchlamys, Tithonia diversifolia, Trichanthera gigantea, Hibiscus rosa sinensis and Mussaenda Alicia, rooting systemic, organic and fertilizers that improve gene expression were used, while they accelerate plant phenology, this in order to get bigger and better productions. Composting 60 days of preparation was used with a good percentage of biomass and organic matter as substrate for filling polyethylene bags $25 \times 30 \mathrm{~cm}$ with a capacity of $1 \mathrm{~kg}$. Moreover a strainer was used to minimize large particles that may interrupt the development of the root. 350 stakes of $40 \mathrm{~cm}$ distributed in a complete block design was planted randomly, separated into five groups each species with 70 stakes. Treatments were T0 (control without application of enraizador), T1 (vinegar), T2 (Hormonagro), T3 (aloe vera) and T4 (phosphate rock), assessments are performed at 15, 30. 45, 60. 75, 90 and 105 days, of the following variables: plant height $(\mathrm{cm})$, diameter of the stem of the plant $(\mathrm{cm})$, root weight $(\mathrm{g})$ and number thereof. Tithonia diversifolia growth was higher $(\mathrm{P}<0.05)$ in all treatments compared with other forage, being greater length in the control $(65.55 \mathrm{~cm})$, T0 difference of more than $20 \mathrm{~cm}$ was observed regarding the treatments used products. The increase in length after 15 days of sowing Tithonia diversifolia in T0 was $15 \mathrm{~cm}$, reaching $35 \mathrm{~cm}$ at 30 and gradually increasing to $65.5 \mathrm{~cm}$ at 105 days, while the others under the same age, are less than $30 \mathrm{~cm}$, showing a similar and slow growth.

Keywords: Legumes, forage, root development. 


\section{RESUMO}

Este trabalho foi desenvolvido na Universidade do Llanos, Barcelona sede km 12 via Puerto López, onde cinco espécies foram plantadas no viveiro Megakespasma erytronchlamys, Tithonia diversifolia, Trichanthera gigantea, Hibiscus rosa sinensis y Mussaenda alicia, fertilizantes orgânicos sistêmicos e de enraizamento que melhoram a expressão de genes foram usados, enquanto acelerar fenologia da planta, esta, a fim de obter produções maiores e melhores. Compostagem de 60 dias após a preparação foi usada com uma boa percentagem de biomassa e de matéria orgânica, como substrato para o enchimento de sacos de polietileno de 25 x $30 \mathrm{~cm}$, com uma capacidade de $1 \mathrm{~kg}$. Além disso, uma peneira foi utilizado para minimizar as partículas grandes que podem interromper o desenvolvimento da raíz. Foi plantada 350 estacas de $40 \mathrm{~cm}$, distribuídos em um delineamento de blocos ao acaso, se separaram em cinco grupos cada espécie, com 70 participações. Os tratamentos foram T0 (testemunha sem aplicação de enraizador), T1 (vinagre), T2 (Hormonagro) T3 (aloe vera) e T4 (rocha fosfática), avaliações são realizadas aos 15, 30. 45, 60. 75, 90 e 105 dias das seguintes variáveis: altura da planta $(\mathrm{cm})$, diâmetro do caule da planta $(\mathrm{cm})$, peso da raiz $(\mathrm{g})$ e do mesmo número. $O$ crescimento Tithonia diversifolia foi maior $(P<0.05)$ em todos os tratamentos em comparação com outra forragem, sendo maior comprimento no controlo $(65.55 \mathrm{~cm})$, T0 diferença de mais de $20 \mathrm{~cm}$ é observado em relação a tratamentos produtos utilizados. O aumento no comprimento após 15 dias da semeadura Tithonia diversifolia T0 era de $15 \mathrm{~cm}$, atingindo $35 \mathrm{~cm}$ a 30 e, gradualmente, aumentando para $65.5 \mathrm{~cm}$ em 105 dias, enquanto os outros sob a mesma idade, estão a menos de $30 \mathrm{~cm}$, mostrando um crescimento semelhante e lento.

Palavras-chave: Leguminosas, forragem, desenvolvimento radicular.

\section{INTRODUCCIÓN}

Los arboles forrajeros en sistemas productivos se utilizan para sombra, ramoneo, corte y suministro en la alimentación de animales (Gallego et al., 2012), pero 
tienen dos limitantes, uno de tipo biológico y el otro económico, el primero establece el tiempo de oferta de biomasa después del corte, cuando se cosecha, y el segundo el costo de la mano de obra para las podas, es por esto, que se hace necesario analizar la eficiencia biológica y calidad nutricional en las diferentes etapas de desarrollo de la planta, lo cual se puede realizar a través del seguimiento agronómico, estableciendo el tiempo en el cual la arbustiva tiene las mejores condiciones que puedan beneficiar a los animales y por tanto a sus factores productivos (Sánchez y Rosales, 2003).

Por otro lado, al iniciar el establecimiento de forrajes en vivero, se permite prevenir y controlar los efectos de depredadores y enfermedades que dañan las plántulas en su etapa de mayor vulnerabilidad, gracias a que se proporciona los cuidados necesarios y las condiciones propicias para lograr un buen desarrollo, por lo tanto las semillas y las plántulas tienen mayores probabilidades de sobrevivencia y adaptación cuando son trasplantadas a su lugar definitivo (Boby y Valdivia, 2005).

El uso de herbicidas orgánicos, enraizadores, fertilizantes son suplementos que se le añade a las plantas, tiene como finalidad fortalecer las plantas y sus raíces, si bien no son indispensables, pueden llegar a ser muy útiles en determinadas circunstancias. Los enraizadores son productos a base de hormonas vegetales naturales $u$ otros compuestos, que estimulan el crecimiento de raíces en estacas, esquejes, brotes o gajos con él tratados. Son importantes complementos que aseguran el crecimiento radicular en todo tipo de vegetales (Azcon y Talon, 2000).

Una de las alternativas para tener mayor éxito en el prendimiento de las partes vegetativas son los enraizadores, ya que ayudan a la proliferación y formación de un buen sistema radicular que permite el crecimiento y desarrollo de una nueva planta, la formación de las raíces es vital para absorber y conducir agua y minerales disueltos, acumular nutrientes y sujetar la planta al suelo. El uso de fitohormonas que aceleran o favorecen el enraizamiento de los esquejes, puede cubrir la necesidad de producción del material vegetativo. Se puede recurrir al empleo de auxinas sintéticas del mismo tipo que produce la planta de manera natural en los brotes terminales y al abrirse las yemas, estas auxinas estimulan la 
formación de raicillas, la falta de suficiente producción de hormonas se completa con estimulantes artificiales tales como el ácido indolbutirico (IBA) y el ácido naftalenoacetico (ANA), los mismos que pueden ser aplicados en solución o polvo o en forma pastosa, las cantidades a emplearse son muy pequeñas pues si se excede se ocasionan serios daños (Lema, 2012)

Este trabajo se ejecutó en el pie de monte llanero, con climas de dos épocas alternantes (lluvia y verano) donde los suelos presentan un alto índice de acidez; las forrajeras utilizadas fueron: Trichanthera gigantea (nacedero), Hibiscus rosasinensis (cayeno), Megakespasma erytrachlamys (camarón rojo), Mussaenda alicia (musaenda rosa) y Tithonia diversifolia (botón de oro), las cuales fueron sembradas en vivero, utilizando enraizadores sistémicos, orgánicos y fertilizantes que mejoran la expresión genética, a la vez que aceleran la fenología de la planta, esto con el fin de obtener mejores y mayores producciones.

\section{METODOLOGÍA}

El experimento se realizó en la Granja Barcelona, adscrita a la Facultad de Ciencias Agropecuarias y Recursos Naturales de la Universidad de los Llanos en el departamento del Meta, vereda Barcelona, km 12 vía Puerto López, ubicada en Villavicencio, a $467 \mathrm{msnm}$ con una temperatura media de $28^{\circ} \mathrm{C}$ y una precipitación media anual de $4.359 \mathrm{~mm}$ (humedad relativa promedio anual de $82 \%$ ), $21.5^{\circ} \mathrm{C}$ de punto de rocío, 1623.8 horas/sol, $1408.6 \mathrm{~mm}$ de evapotranspiración, $1.9 \mathrm{~m} / \mathrm{seg}$ de velocidad de viento (IDEAM, 2014).

En la etapa de vivero, que tuvo una duración de cuatro meses, fue fundamental las condiciones suministradas al material de crecimiento de la planta, su adecuación, el ambiente y manejo de labranza, puesto que los riesgos ambientales a los que está expuesta la planta en esta etapa son reducidos, es una ventaja el control que se tiene sobre el material sembrado.

Se registraron secuencialmente los datos, con lo cual se estableció el análisis fenológico y radicular de las especies forrajeras arbustivas: Trichanthera gigantea (nacedero), Hibiscus rosa-sinensis (cayeno), Tithonia diversifolia (botón de oro), 
Megakespasma erytrachlamys (camarón rojo) y Mussaenda alicia (musaenda rosa). El vivero donde se sembraron estas especies fue encerrado con polietileno y se utilizó un sistema de labranza mínima. Para evitar problemas de sanidad y minimizar riesgos, se hizo una exhaustiva limpieza para la adecuación del terreno, librando de toda especie arvense que pudiese repercutir con la actividad realizada.

Los sustratos utilizados para la siembra de cualquier especie de planta son de carácter prioritario, debido a que el suministro nutricional de la raíz es fundamental, por lo tanto se utilizó compostaje de 60 días de preparado con buen porcentaje de biomasa y materia orgánica, como sustrato para el llenado de las bolsa de un $\mathrm{kg}$ en donde fueron sembradas las especies arbustivas forrajeras mencionadas anteriormente. El compostaje fue pasado por un zarando, para minimizar partículas grandes que pudieran interrumpir el desarrollo de la raíz. Se sembraron 350 estacas de $40 \mathrm{~cm}$ de longitud, en bolsas de polietileno de $25 \mathrm{~cm}$ de ancho por $30 \mathrm{~cm}$ de largo, en un diseño completamente al azar, se separaron en cinco grupos de 70 estacas, cada uno por especie de planta, divididos en siete estacas por cinco tratamientos con dos repeticiones. Los tratamientos fueron los siguientes: T0 (testigo, sin aplicación), T1 (vinagre), T2 (hormonagro, ANA), T3 (aloe vera) y T4 (roca fosfórica); la aplicación de estos compuestos se realizó en el extremo del material que se va a sembrar, excepto la roca fosfórica que se incluyó en la tierra. Se evaluaron a los 15, 30. 45, 60. 75, 90 y 105 días, las siguientes variables: altura de la planta $(\mathrm{cm})$, diámetro de la planta $(\mathrm{cm})$, peso de las raíces (gr) y número de las mismas. Todas las plantas se mantuvieron en el mismo vivero, protegidas con polisombra para evitar daños por exceso de sol o de lluvia; además el vivero fue controlado y encerrado en su totalidad para no tener problemas de agentes externos, factores que fueron controlados para obtener un $100 \%$ de prendimiento en todos los tratamientos (Figura 1).

T1 = Adición de vinagre, este compuesto de sabor y olor agrio, siendo su fórmula química $\mathrm{CH}_{3}-\mathrm{OOH}\left(\mathrm{C}_{2} \mathrm{H}_{4} \mathrm{O}_{2}\right)$, también se le denomina sistemáticamente ácido etanoico. Este producto mientras sea derivado de productos naturales y no de químicos, es aceptable dentro de la agricultura orgánica para ser utilizado como 
un herbicida orgánico. El ácido acético se degrada rápidamente en el agua (es por eso que no es recomendable aplicarlo luego de una lluvia) y no se bioacumula. Si bien es cierto que el vinagre reduce el $\mathrm{pH}$ del suelo hay que tener en cuenta que este valor volverá a su estado normal dentro de 48 horas, además, el vinagre es un producto biodegradable (Organic, 2008).

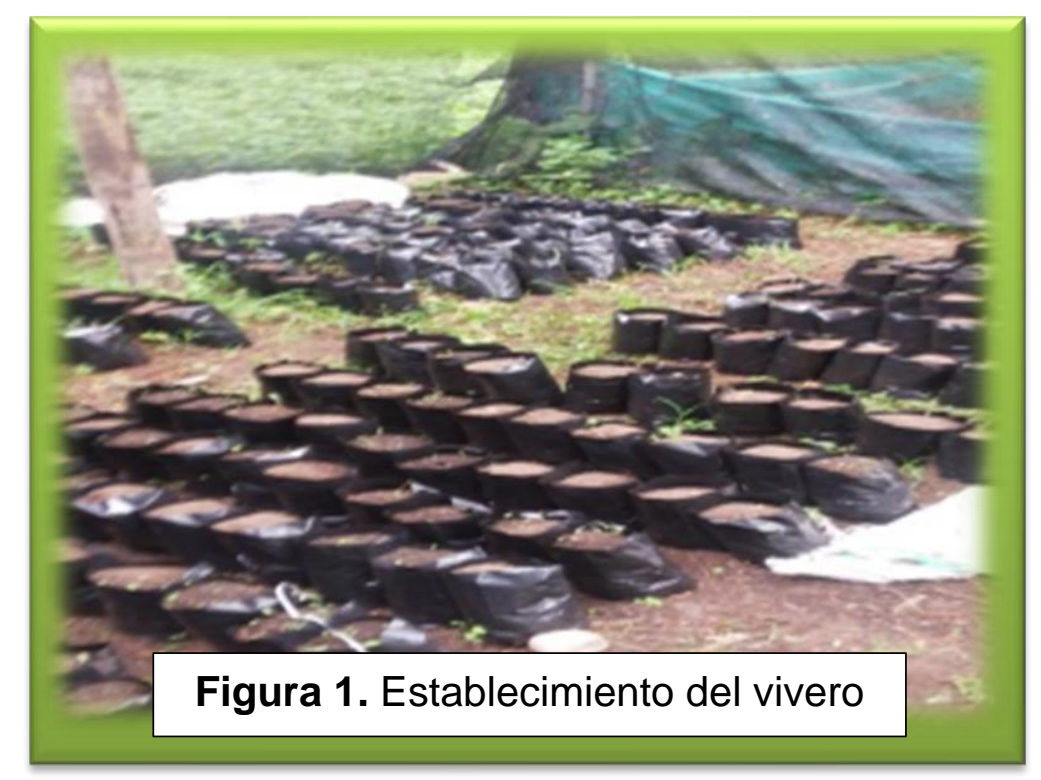

T2 = Se adicionó hormonagro, el cual es un enraizador químico compuesto por alfa-naftalenacético al $0.4 \%$ (ANA), al parecer, debido a que no es destruido por enzimas en el suelo, persiste más tiempo en el sustrato. Del mismo modo, promueve la elongación de secciones escindidas de raíces e incluso de raíces intactas de muchas especies, aumentando rápidamente su velocidad de crecimiento. Esta hormona estimula el desarrollo de raíces y puede combinarse con otras sustancias para estimular la formación de brotes y raíces (Taiz y Zeiger, 2006).

T3 = Se adicionó aloe vera, en varios estudios han demostrado efectos estimulantes en el crecimiento con el extracto de gel de aloe vera, particularmente con relación a la formación de raíces, superando incluso a los reguladores químicos utilizados tradicionalmente, debido a que la actividad de la hormona auxina presente en el aloe estimula el crecimiento y enraizamiento en las plántulas 
de plátano y orquídeas en dosis del 2, 4 y 6 \% (Rodríguez y Hechevarria, 2006; Jo et al., 2008).

T4 = Se adicionó roca fosfórica (pentóxido de fósforo, $\mathrm{P}_{2} \mathrm{O}_{5}$ ), puesto que el fósforo es un elemento nutritivo esencial para las raíces, y su deficiencia reduce severamente los rendimientos de los cultivos, particularmente en suelos ácidos de zonas tropicales y subtropicales, es necesario aplicar cantidades importantes de insumos fosfóricos para obtener un crecimiento óptimo de los cultivos y forrajes. A pesar de tener una composición extremadamente variable, son la fuente principal de fósforo para la fabricación de fertilizantes fosfatados y otros productos químicos (Chien et al., 2003; Singh et al., 2003).

El diseño estadístico fue bloques completos al azar y su modelo matemático el siguiente: $Y i j=\mu+T i+\beta j+\varepsilon i j$. Donde, $Y i j=$ Observación en la variable experimental (altura de la planta, diámetro del tallo, peso y número de raíces); $\mu$ = Parámetro, efecto medio; $T i=$ Parámetro, efecto del tratamiento $i($ T0. T1, T2, T3 y T4), $\beta j=$ Parámetro, efecto del bloque j (las cinco forrajeras); $\varepsilon i j=$ Valor aleatorio, error experimental de la unidad ij.

La variables analizadas fueron: longitud, diámetro, peso y número de la raíces, a las cuales se realizó análisis de varianza y se hicieron las pruebas post-hoc para comparar las medias de cada tratamiento y bloque, utilizando la prueba de Duncan, en programa estadístico SPSS V.19.

\section{RESULTADOS Y DISCUSIÓN}

\section{Crecimiento de las plantas}

A los 105 días el cultivo de botón de oro sin tratamientos (testigo), presentó el mejor crecimiento $(\mathrm{P}<0.05)$ de las cinco especies evaluadas, seguido de nacedero, sin presentar diferencias con cayeno y camarón rojo, siendo la de menor longitud musaenda $(\mathrm{P}<0.05)$, en comparación con las otras tres especies forrajeras (Tabla1). 
El crecimiento del botón de oro fue superior $(P<0.05)$ en todos los tratamientos en comparación con las otras forrajeras, siendo mayor su longitud en el testigo (65,55 $\mathrm{cm})$. En T0 se observa diferencia de más de $20 \mathrm{~cm}$ con relación a los tratamientos que utilizaron enrraizadores (Tabla 1), esto significa que el botón de oro no requiere de estos compuestos para su crecimiento en vivero, lo único a tener en cuenta, es que la tierra en la bolsa tenga suficiente materia orgánica. El aumento de longitud a los 15 días de sembrado el botón de oro en T0 fue de $15 \mathrm{~cm}$, alcanzando $35 \mathrm{~cm}$ a los 30 e incrementando gradualmente hasta los $65.5 \mathrm{~cm}$ a los 105 días mientras las otras especies a esta misma edad días medían menos de 30 $\mathrm{cm}$, demostrando un crecimiento similar y lento (Grafica 1 y Tabla 1).

Tabla 1. Crecimiento (longitud cm) de las arbóreas a los 105 días de evaluación

\begin{tabular}{cccccc}
\hline \multirow{2}{*}{ Forrajes } & \multicolumn{5}{c}{ Tratamientos } \\
\cline { 2 - 6 } & $\begin{array}{c}\text { Sin } \\
\text { Enraizador }\end{array}$ & Vinagre & $\begin{array}{c}\text { Hormoagro } \\
\text { (Ana) }\end{array}$ & Aloe Vera & $\begin{array}{c}\text { Roca } \\
\text { Fosfórica }\end{array}$ \\
\hline Nacedero & $24.07^{\mathrm{bB}}$ & $26.43^{\mathrm{bB}}$ & $17.07^{\mathrm{aB}}$ & $18.00^{\mathrm{aA}}$ & $22.14^{\mathrm{abB}}$ \\
Cayeno & $18.29^{\mathrm{aB}}$ & $35.00^{\mathrm{bC}}$ & $23.43^{\mathrm{aB}}$ & $16.00^{\mathrm{aA}}$ & $26.29^{\mathrm{abB}}$ \\
Camarón rojo & $20.86^{\mathrm{aB}}$ & $20.57^{\mathrm{aB}}$ & $23.31^{\mathrm{aB}}$ & $24.86^{\mathrm{aB}}$ & $29.86^{\mathrm{bB}}$ \\
Musaenda rosa & $5.26^{\mathrm{aA}}$ & $8.71^{\mathrm{abA}}$ & $5.43^{\mathrm{aA}}$ & $12.71^{\mathrm{bA}}$ & $5.26^{\mathrm{aA}}$ \\
Botón de oro & $65.50^{\mathrm{bC}}$ & $35.42^{\mathrm{aC}}$ & $30.60^{\mathrm{aC}}$ & $40.00^{\mathrm{aC}}$ & $41.00^{\mathrm{aC}}$ \\
\hline
\end{tabular}

Letras minúsculas distintas en la misma fila. los tratamientos son distintos $(P<0.05)$.

Letras mayúsculas distintas en la misma columna. existe diferencia entre las forrajeras $(P<0.05)$.

El comportamiento del nacedero fue mejor $(\mathrm{P}<0.05)$ en el testigo y cuando se adicionó vinagre y roca fosfórica, observándose, que la adición de vinagre (T1), puede mejorar levemente su crecimiento puesto que su longitud llego máximo a $26.43 \mathrm{~cm}$, siendo similar a la de botón de oro y cayeno, que obtuvo su mayor con crecimiento con $\mathrm{T} 1$, siendo superior a su vez $(\mathrm{P}<0.05)$ a la de camarón rojo y musaenda (Tabla 1 y Gráfica 2). 


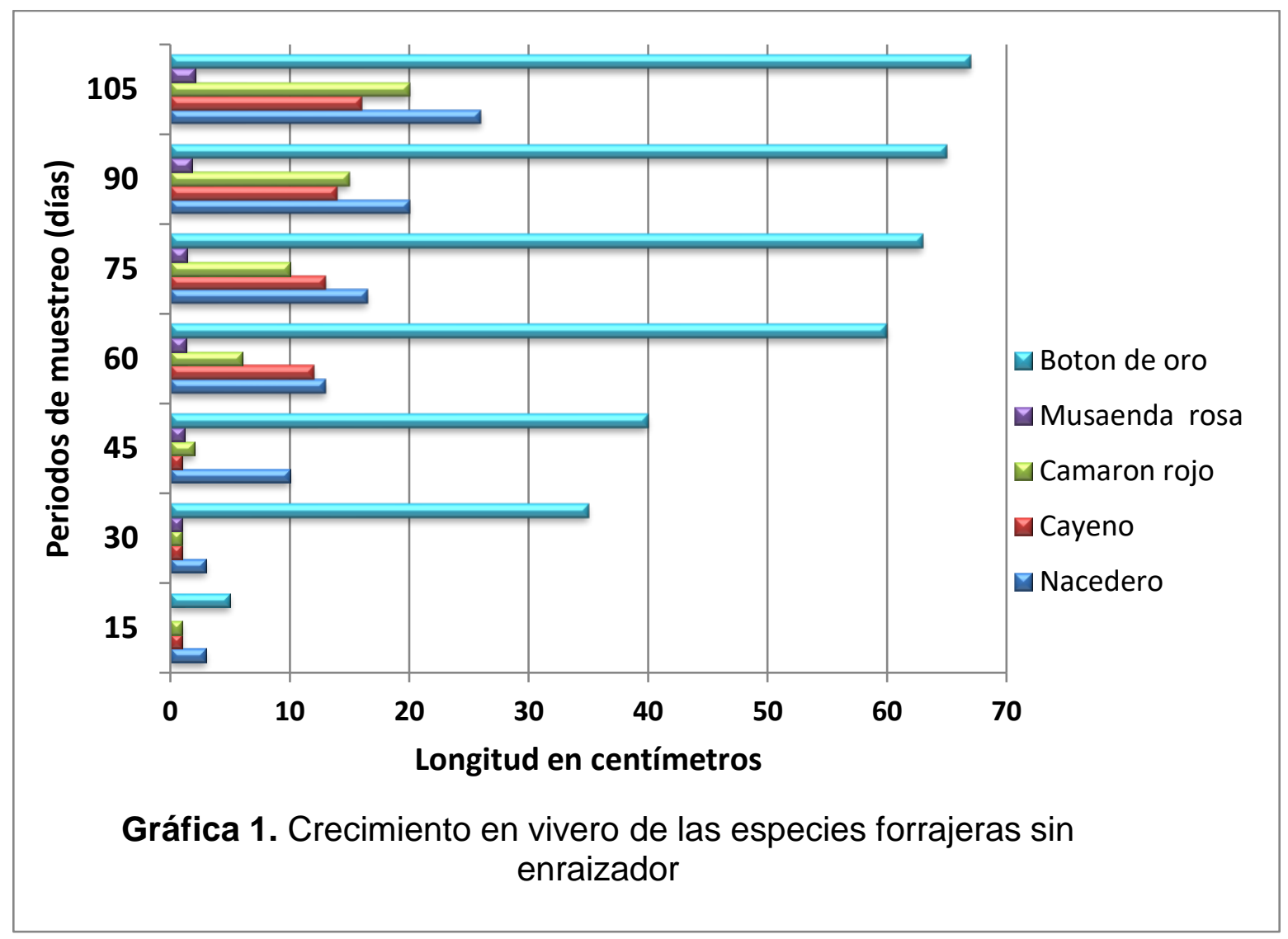

En el tratamiento con hormonagro, se observó que musaenda rosa fue la de menor crecimiento $(P<0.05)(5.43 \mathrm{~cm})$, mientras que nacedero, cayeno y camarón presentaron un comportamiento similar con T3 (Tabla 1 y Grafica 3), posiblemente este compuesto estimuló el crecimiento de su sistema radicular de estas tres especies, lo cual es muy importante porque las hormonas (ANA) aseguran el desarrollo y fortalecimiento del sistema radicular en todo tipo de vegetales (Azcón y Talón, 2000). Además las raíces son vitales para absorber y conducir agua y minerales disueltos, acumular nutrientes y sujetar la planta al suelo, cuando son trasplantadas al sitio definitivo (Lema, 2012).

Se observa, que la musaenda rosa respondió mejor $(\mathrm{P}<0.05)$ a la adición de aloe vera (T3) en comparación con los demás tratamientos, aunque, si se hace la comparación con las demás especies su respuesta fue menor $(P<0.05)(12.71 \mathrm{~cm})$ con relación a nacedero $(18.0 \mathrm{~cm})$, cayeno $(16.0 \mathrm{~cm})$, camarón rojo $(24.86 \mathrm{~cm})$ y botón de oro $(40.0 \mathrm{~cm})$, (Tabla 1 y Grafica 4$)$, en investigaciones se ha 
demostrado que el aloe vera influye de manera positiva en la formación de raíces, superando incluso a los reguladores químicos utilizados tradicionalmente, debido a la actividad de su hormona auxina (Rodríguez y Hechevarria, 2006; Jo et al., 2008).

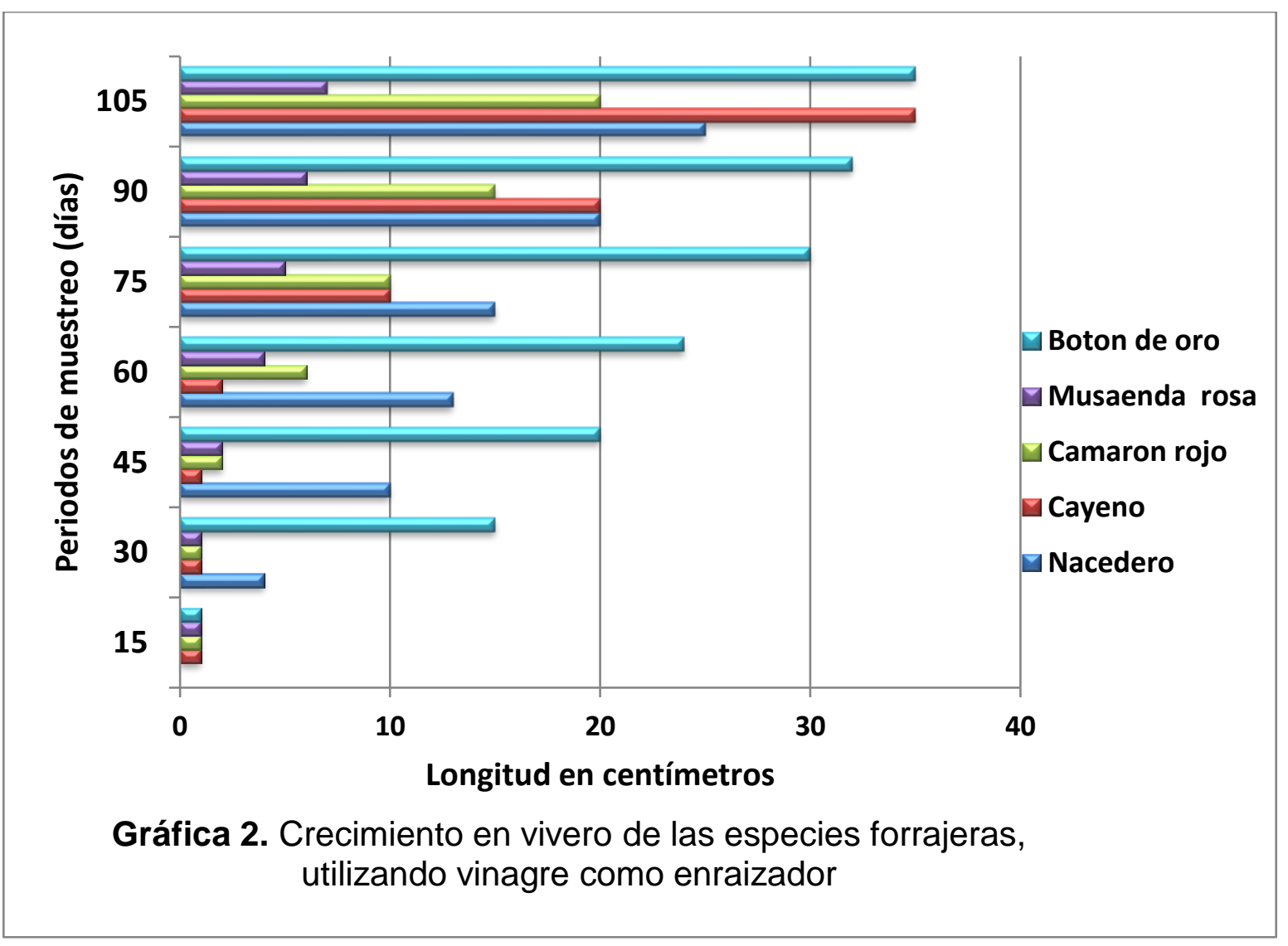

El crecimiento del camarón rojo fue mayor $(\mathrm{P}<0.05)$ cuando se adicionó roca fosfórica $(29.86 \mathrm{~cm})$ con respecto a los otros tratamientos, aunque su comportamiento fue similar al de nacedero $(22.14 \mathrm{~cm})$ y cayeno $(26.29)$, siendo el de menor longitud musaenda $(5.26 \mathrm{~cm})$, es de anotar que el cayeno mostró mejor crecimiento cuando se adicionó vinagre y roca fosfórica (Tabla 1 y Gráficas 2 y 5).

La buena respuesta del cayeno a la adición de vinagre y roca fosfórica puede estar relacionada con el primero, que es considerado en la agricultura como un herbicida orgánico, aunque este compuesto reduce el $\mathrm{pH}$ del suelo hay que tener en cuenta que este valor volverá a su estado normal dentro en 48 horas, porque es un producto de alta solubilidad en el agua por lo tanto es biodegradable 
(Organic, 2008). Referente al segundo, la roca fosfórica, a pesar de tener una composición muy variable, es una fuente importante de fósforo, lo cual es beneficioso, puesto que los suelos de la zona del Piedemonte llanero son deficientes en este mineral, que es fundamental para el crecimiento óptimo de los cultivos y forrajes. Además la roca fosfórica es el insumo para la fabricación de fertilizantes fosfatados y otros compuestos químicos que se utilizan en las plantas para mejorar su producción (Chien et al., 2003; Singh et al., 2003).

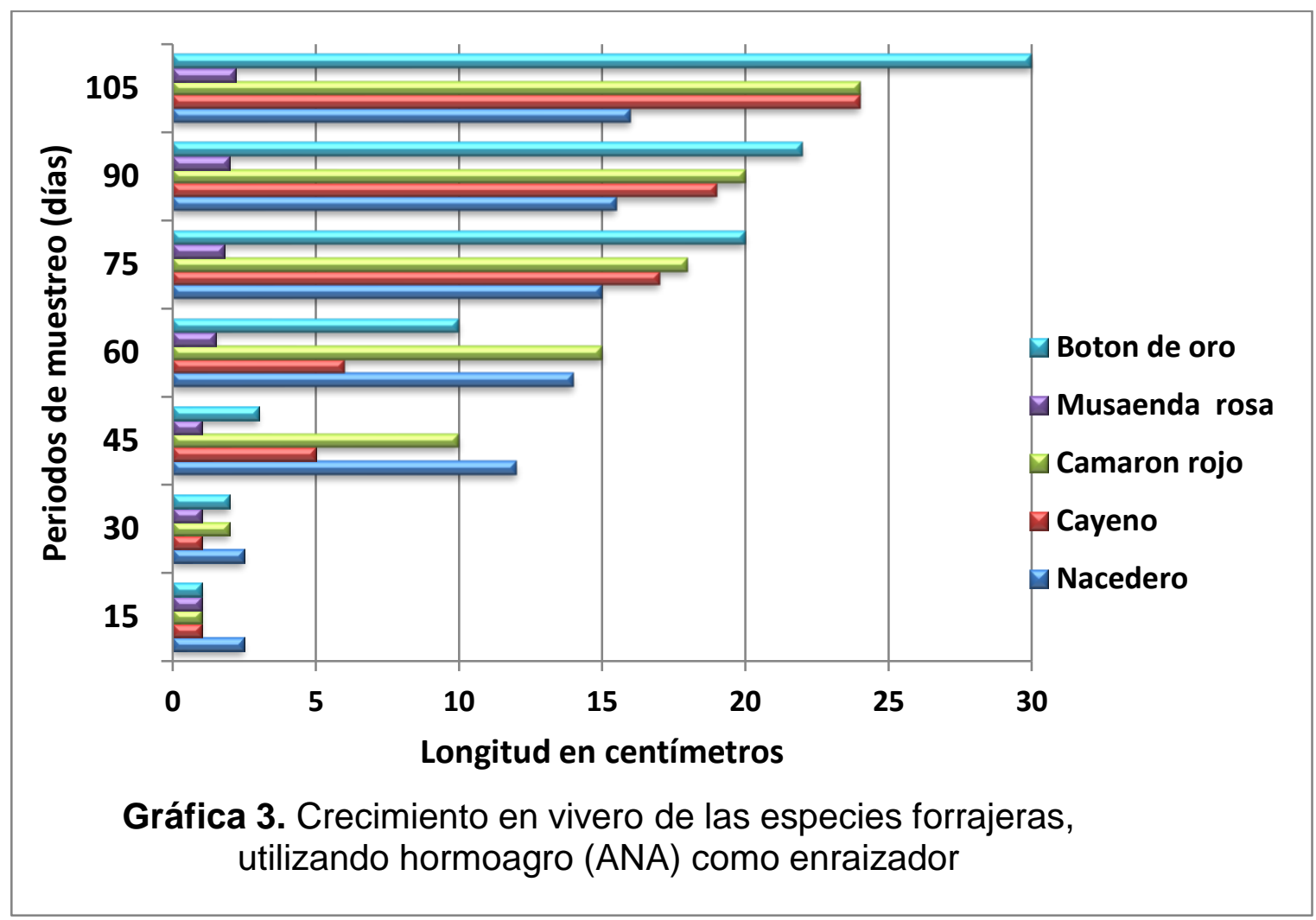

\section{Diámetro del tallo}

El diámetro del botón de oro fue mayor $(\mathrm{P}<0.05)$ en todos los tratamientos en comparación con las demás especies, aunque la respuesta de esta forrajera fue similar en todos los tratamientos (Tabla 2), se observó un mayor diámetro (2.71 $\mathrm{cm}$ ) cuando se adicionó hormonagro. Comportamiento similar al botón de oro en lo referente al grosor del tallo, lo presentaron nacedero y cayeno cuando se les adicionó hormonagro, aloe vera y roca fosfórica: para la primera especie 2.93, 1.73 y $2.06 \mathrm{~cm}$, para la segunda $2.07,2.11$ y $2.26 \mathrm{~cm}$ respectivamente (Tabla 2). 

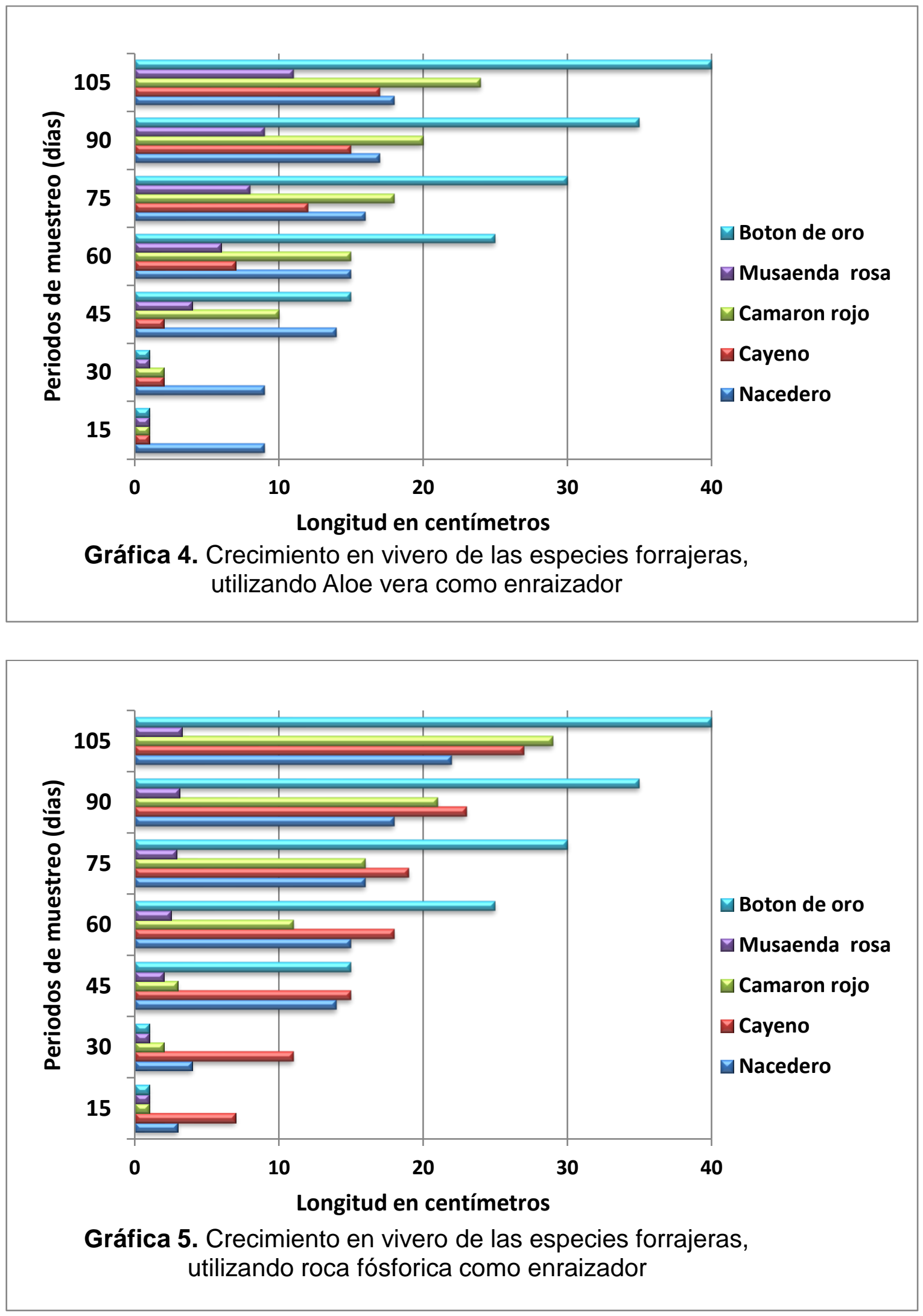
Tabla 2. Promedio de diámetro $(\mathrm{cm})$ de las arbóreas a los 105 días de evaluación

\begin{tabular}{cccccc}
\hline \multirow{2}{*}{ Forrajes } & \multicolumn{5}{c}{ Tratamientos } \\
\cline { 2 - 6 } & $\begin{array}{c}\text { Sin } \\
\text { Enraizador }\end{array}$ & Vinagre & $\begin{array}{c}\text { Hormoagro } \\
\text { (Ana) }\end{array}$ & $\begin{array}{c}\text { Aloe } \\
\text { Vera }\end{array}$ & $\begin{array}{c}\text { Roca } \\
\text { Fosfórica }\end{array}$ \\
\hline Nacedero & $1,50^{\mathrm{aA}}$ & $1,89^{\mathrm{aB}}$ & $2,93^{\mathrm{bB}}$ & $1,73^{\mathrm{aAB}}$ & $2,06^{\mathrm{aAB}}$ \\
Cayeno & $1,11^{\mathrm{aA}}$ & $1,29^{\mathrm{abA}}$ & $2,07^{\mathrm{bAB}}$ & $2,11^{\mathrm{bB}}$ & $2,26^{\mathrm{CB}}$ \\
Camarón rojo & $1,67^{\mathrm{aA}}$ & $1,69^{\mathrm{aAB}}$ & $1,68^{\mathrm{aA}}$ & $1,66^{\mathrm{aA}}$ & $1,33^{\mathrm{aA}}$ \\
Musaenda rosa & $1,26^{\mathrm{abA}}$ & $1,34^{\mathrm{abA}}$ & $1,50^{\mathrm{bA}}$ & $0.79^{\mathrm{aA}}$ & $1,54^{\mathrm{abA}}$ \\
Botón de oro & $2,14^{\mathrm{aB}}$ & $2,71^{\mathrm{aC}}$ & $2,21^{\mathrm{aAB}}$ & $2,47^{\mathrm{aB}}$ & $2,48^{\mathrm{aB}}$ \\
\hline
\end{tabular}

Letras minúsculas distintas en la misma fila, los tratamientos son distintos $(P<0.05)$.

Letras mayúsculas distintas en la misma columna, existe diferencia entre las forrajeras $(P<0.05)$.

De acuerdo a lo resultados expuestos, los mayores diámetros de las forrajeras estudiadas, se observaron cuando se empleó fitohormonas contenidas en el ANA, auxinas en aloe vera y el fertilizante como la roca fosfórica que proporciona fósforo $(P)$, nutriente fundamental para el desarrollo de las plantas, Estas sustancias estimulan la planta para producir reacciones naturales, que permiten la formación de brotes y yemas, lo cual está condicionado al desarrollo y fortaleza del tallo, además se requieren pequeñas cantidades, puesto que pueden ocasionar serios daños cuando se utilizan en gran proporción, lo cual ha sido estudiado por Lema, (2012); Rodríguez y Hechevarria, (2006); Jo et al, (2008).

\section{Peso y número de raíces}

El cultivo de botón de oro presentó el mayor peso de sus raíces $(P<0.05)$ con hormonagro, además se observó mayor número de raíces en este tratamiento, aunque fue similar en todos los tratamientos, además, presentó el mejor desarrollo radicular $(\mathrm{P}<0.05)$ en comparación con las otras especies evaluadas (Tablas 3 y 4). También, se observó que las plántulas del botón de oro presentaron gran cantidad de hojas al final de la etapa de vivero, mostrando buena textura y una serie de raíces indefinidas, rígidas y de gran agresividad lo que indica una madurez radicular temprana. Estas raíces profundizaron y rompieron las bolsas a 
los 60 días de sembradas, este factor puede dificultar el manejo en vivero debido a su desarrollo tan acelerado, por lo tanto se recomienda hacer un trasplante al sitio definitivo máximo a los 60 días.

Tabla 3. Promedio de peso de la raíz (gr) las arbóreas a los 105 días de evaluación

\begin{tabular}{cccccc}
\hline \multirow{2}{*}{ Forrajes } & \multicolumn{5}{c}{ Tratamientos } \\
\cline { 2 - 6 } & $\begin{array}{c}\text { Sin } \\
\text { Enraizador }\end{array}$ & Vinagre & $\begin{array}{c}\text { Hormoagro } \\
\text { (Ana) }\end{array}$ & $\begin{array}{c}\text { Aloe } \\
\text { Vera }\end{array}$ & $\begin{array}{c}\text { Roca } \\
\text { Fosfórica }\end{array}$ \\
\hline Nacedero & $0.61^{\mathrm{aA}}$ & $1,59^{\mathrm{abB}}$ & $1,33^{\mathrm{abA}}$ & $1,94^{\mathrm{bAB}}$ & $2,94^{\mathrm{cB}}$ \\
Cayeno & $0.51^{\mathrm{aA}}$ & $1,41^{\mathrm{abAB}}$ & $2,13^{\mathrm{bA}}$ & $1,09^{\mathrm{abA}}$ & $0.31^{\mathrm{aA}}$ \\
Camarón rojo & $3,71^{\mathrm{bB}}$ & $4,50^{\mathrm{bC}}$ & $0.46^{\mathrm{aA}}$ & $2,29^{\mathrm{abB}}$ & $3,07^{\mathrm{abB}}$ \\
Musaenda rosa & $2,01^{\mathrm{bA}}$ & $0.77^{\mathrm{aA}}$ & $0.94^{\mathrm{aA}}$ & $1,03^{\mathrm{aA}}$ & $0.70^{\mathrm{aA}}$ \\
Botón de oro & $4,75^{\mathrm{aC}}$ & $3,21^{\mathrm{aBC}}$ & $7,00^{\mathrm{bB}}$ & $5,43^{\mathrm{aC}}$ & $5,47^{\mathrm{aC}}$ \\
\hline
\end{tabular}

Letras minúsculas distintas en la misma fila, los tratamientos son distintos $(P<0.05)$.

Letras mayúsculas distintas en la misma columna, existe diferencia entre las forrajeras $(P<0.05)$.

Tabla 4. Promedio de numero de raíces de las arbóreas a los 105 días de evaluación

\begin{tabular}{cccccc}
\hline \multirow{2}{*}{ Forrajes } & \multicolumn{5}{c}{ Tratamientos } \\
\cline { 2 - 6 } & $\begin{array}{c}\text { Sin } \\
\text { Enraizador }\end{array}$ & Vinagre & $\begin{array}{c}\text { Hormoagro } \\
\text { (Ana) }\end{array}$ & $\begin{array}{c}\text { Aloe } \\
\text { Vera }\end{array}$ & $\begin{array}{c}\text { Roca } \\
\text { Fosfórica }\end{array}$ \\
\hline Nacedero & $12^{\mathrm{aB}}$ & $14^{\mathrm{aB}}$ & $55^{\mathrm{cC}}$ & $22^{\mathrm{abB}}$ & $17^{\mathrm{abB}}$ \\
Cayeno & $2^{\mathrm{aA}}$ & $2^{\mathrm{aA}}$ & $21^{\mathrm{bB}}$ & $3^{\mathrm{aA}}$ & $8^{\mathrm{aA}}$ \\
Camarón rojo & $10^{\mathrm{abA}}$ & $7^{\mathrm{aA}}$ & $2^{\mathrm{aA}}$ & $18^{\mathrm{bB}}$ & $7^{\mathrm{aA}}$ \\
Musaenda rosa & $2^{\mathrm{aA}}$ & $4^{\mathrm{aA}}$ & $3^{\mathrm{aA}}$ & $2^{\mathrm{aA}}$ & $4^{\mathrm{aA}}$ \\
Botón de oro & $7^{\mathrm{aA}}$ & $6^{\mathrm{aA}}$ & $15^{\mathrm{aAB}}$ & $9^{\mathrm{aA}}$ & $9^{\mathrm{aA}}$ \\
\hline
\end{tabular}

Letras minúsculas distintas en la misma fila, los tratamientos son distintos $(P<0.05)$.

Letras mayúsculas distintas en la misma columna, existe diferencia entre las forrajeras $(P<0.05)$.

Los resultados demuestran un buen desarrollo radicular del botón de oro en este experimento, los cuales se pueden confirmar con los experimentos realizados por Uribe et al., (2011), en los cuales se evaluaron el número de raíces y porcentaje 
de prendimiento a los 15 días después de la siembra en vivero, encontrando un $94 \%$ de prendimiento en estacas y un número de raíces fue de 4.25 .

En el comportamiento del nacedero se puede observar un mayor peso de la raíz con roca fosfórica $(\mathrm{P}<0.05) 2.94 \mathrm{~g}$, aunque su número de raíces (22) es menor $(\mathrm{P}<0.05)$ en comparación con el tratamiento con hormonagro (55), en el cual su peso fue inferior $1.33 \mathrm{~g}$, de esto se deduce que cuando el nacedero desarrolla mayor número de raíces, su peso es menor y viceversa (Tablas 3 y 4), también se observó que el nacedero es una especie que se enfatiza primero en desarrollar su morfología radicular antes que la foliación. La relación inversa del peso con número de raíces se confirma, al comparar el nacedero con otras especies, en el testigo el peso de raíz fue similar, exceptuando el botón de oro, aunque su número de raíces fue superior $(\mathrm{P}<0.05)$ con relación a las otras especies. En el tratamiento con vinagre el nacedero y cayeno fueron similares y superiores en el peso de la raíz en comparación con musaenda, mientras que el número de raíces del nacedero fue superior $(\mathrm{P}<0.05)$ con relación a las otras especies (Tablas 3 y 4 ). En estudios realizados por Giraldo et al., (2009) quienes trabajaron con nacedero, midiendo el peso de la raíz a los 60 días, observaron una mejor respuesta con ANA $(0.69 \mathrm{~g})$ en comparación con aloe vera $(0.53 \mathrm{~g})$, mientras que en este experimento se observó una mejor respuesta con aloe vera en relación al ANA a los 105 días (1.94 Vs $1.33 \mathrm{~g}$, respectivamente).

El cayeno en su periodo inicial sin la utilización de ningún material o estimulante (testigo) presentó una emisión de la raíz a los 60 días su peso $(2.13 \mathrm{~g})$ y número de raíces (21), a los 105 días fue mayor $(P<0.05)$ cuando se utilizó $A N A$, en comparación con los otros tratamientos (Tablas 3 y 4), su emisión de hojas fue rápida, observándose que es una planta resistente a los cambios bruscos de humedad. Por otro lado, el tratamiento de las raíces de cayeno con aloe vera presentó un curtimiento de la peridermis manteniéndose un desarrollo radicular lento.

El desarrollo radicular del camarón rojo inicialmente fue lento hasta los 45 días, sus raíces son cilíndricas y en ocasiones presentaron puntos rosados, el 
tratamiento con vinagre presentó el mayor $(\mathrm{P}<0.05)$ peso de la raíz $(4.5 \mathrm{~g})$, siendo similar con botón de oro $(3.21 \mathrm{~g})$, se observa que en el tratamiento con ANA, el peso $(0.46 \mathrm{~g})$ y número de raíces $(2)$, fue inferior $(P<0.05)$ al testigo (Tablas 3 y 4 ), lo que implica que el uso de fitohormonas no es recomendable para esta planta, aunque su desarrollo fue lento, sus raíces son agresivas y generaron un excelente agarre radicular, con una alta producción de hojas, lo que implica hacia el futuro una gran cantidad de biomasa, siempre que se siembre en un suelo con alto contenido de materia orgánica.

Musaenda rosa es de tipo leñoso por lo tanto emite un sistema radicular pivotante, de crecimiento tardío y comienza a emitir sus hojas antes del desarrollo de sus raíces, en el testigo se observó, que el peso de la raíz $(2,01 \mathrm{~g})$ fue superior $(\mathrm{P}<0.05)$, aunque su número de raíces fue similar, al compararla con los otros tratamientos (Tablas 1 y 2). La musaenda rosa mostró similar comportamiento ( $P>0.05)$ en el peso de sus raíces $(0.77,0.94,1,03$ y $0.70 \mathrm{~g})$, con vinagre, ANA, aloe vera y roca fosfórica respectivamente; se observó en ella, indicios de secarse en la parte de las yemas cuando se aplicaron los tres primeros componentes.

\section{CONCLUSIONES}

Los componentes aplicados fueron de gran importancia para el crecimiento de las plantas, exceptuando botón de oro que alcanzó su mayor longitud en el tratamiento testigo, siendo el diámetro del tallo similar en todos los tratamientos, su respuesta a la adición de ANA se reflejó en el peso y número de raíces que fueron mayores en comparación con los otros tratamientos. Se observó un comportamiento superior en el botón de oro, en las variables evaluadas con relación a las otras especies.

El cayeno y nacedero respondieron muy bien en su crecimiento, cuando se utilizó vinagre y roca fosfórica, aunque el mayor diámetro del tallo y número de raíces de estas dos especies fue con la adición de ANA, el peso de las raíces del nacedero fue similar al testigo, mientras el cayeno presentó su mayor peso con hormonagro. 
El camarón rojo respondió con buen crecimiento, a la adición de ANA, aloe vera y roca fosfórica, observándose un mayor peso en sus raicees cuando se utilizó este último compuesto. La musaenda presento mayor longitud cuando se le adicionó vinagre y aloe vera, obteniendo el mayor peso de sus raíces con este último producto.

Se comprueba que para el botón de oro no es necesario utilizar ningún tipo de componente químico, puesto que su comportamiento radicular en el testigo fue excelente, se concluye que esta especie es muy buena para establecer sistemas para la suplementación alimenticia, por su gran cantidad de biomasa y desarrollo radicular que se obtiene en corto tiempo.

\section{RECOMENDACIONES}

Analizar los tiempos climáticos y determinar cómo las precipitaciones pueden influir en la investigación, evitando problemas de deshidratación.

Mantener un impecable control de arvenses, evitando una variación de las muestra, y al utilizar el aloe vera, es esencial licuar excelentemente, para obtener un buen suplemento, aprovechando al máximo sus propiedades.

\section{REFERENCIAS BIBLIOGRÁFICAS}

1. Araméndez $\mathrm{H}$, Cardona $\mathrm{C}$, Correa $\mathrm{E}$. Efecto de diferentes sustratos en la calidad de plántulas de berenjena (Solanum melongena L.). Revista Colombiana de Ciencias Hortícolas, 7 (1): 55-61. 2013.

2. Argel PJ, Lascano CE. Cratylia argentea (Desvaux) O. Kuntze: Una nueva leguminosa arbustiva para suelos ácidos en zonas subhúmedas tropicales. Pasturas Tropicales, 20 (1): 37-43. 1998.

3. Azcon J, Talon M. Fundamentos de fisiología vegetal. Ediciones Universitat de Barcelona, primera edición, Barcelona España, p. 286, 287, 317. 2000.

4. Boby FB, Valdivia MA. Evaluación del comportamiento de tres forestales a nivel de vivero en el municipio de Telica, departamento de León. Tesis de Grado, Universidad Nacional Agraria, facultad de Recursos Naturales y del Ambiente, Managua, Nicaragua, 65 p. 2005.

5. Chien SH. Factors affecting the agronomic effectiveness of phosphate rock: a general review. En: S.S.S. Rajan \& S.H. Chien, eds.Direct application of phosphate rock and related technology: latest developments and practical experiences. Proc. Int. Meeting, Kuala Lumpur, 16-20 July 2001. Muscle Shoals, USA, IFDC. 441 p. 2003. 
6. Cruz CJ, Moreno DC. Efecto de la fertilización y diámetro del material vegetativo sobre el crecimiento en vivero de Sambucus nigra y Morus alba, con destino a sistemas silvopastoriles. Tesis de Grado Zootecnista, Universidad de la Salle, Facultad de Zootecnia, 196 p. 2009.

7. Díaz L, Maldonado JC, Peters J, Pérez EM, Puértolas S, Morales D, Jiménez MS, Gil L. Efecto de diferentes sustratos en la calidad de plántulas de berenjena (Solanum melongena L.). Cuad. Soc. Esp. Cien. For. 17: 63-67. 2004.

8. Espinoza JL, Palacios A. La ganadería orgánica, una alternativa de desarrollo pecuario para algunas regiones de México: una revisión. INCI, 32 (6): 385-390. 2007.

9. Gálvez A, Carreño D, Guzmán W. Evaluación en vivero de tres sistemas de reproducción vegetativa de Dahlia imperialis (Roezl ex Ortgies). Revista Investigación Pecuaria, 3 (1): 73-83. 2014.

10. García DE, Medina MG, Cova LJ, Clavero T, Torres A, Perdomo D, Santos O. Evaluación integral de recursos forrajeros para rumiantes en el estado Trujillo, Venezuela, Rev. Fac. Agron., 26 (4): 555-582. 2009.

11. Gallego EJ, Morales S, Vivas N. Propuesta para el uso de especies arbóreas y arbustivas forrajeras en sistemas ganaderos en el valle del Patía, Cauca. Biotecnología en el Sector Agropecuario y Agroindustrial, 10 (2): 207-216. 2012.

12. Hidalgo PR, Sindoni M, Marín C. Evaluación de sustratos a base de vermicompost y enmiendas orgánicas líquidas en la propagación de parchita (Passiflora edulis v. flavicarpa) en vivero. Revista UDO Agrícola, 9 (1): 126135. 2009.

13. Iglesias JM. Sistemas de producción agroforestales. Capacitación y análisis en: "conceptos generales y definiciones". Rev. Sist. Prod. Agroecolo., 2 (1): 151-176. 2011.

14. Instituto de Hidrología, Meteorología y Estudios Ambientales (IDEAM). Información Histórica, Climatografía de las principales ciudades, Cartas Climatologicas - Medias Mensuales, Aeropuerto Vanguardia. 2014. Disponible En: http://bart.ideam.gov.co/cliciu/villao/tabla.htm

15. García M, Hernández R, Echevarria Y, Esteves M, Bustios S. Utilización del aloe vera $L$. en la composición de medios de cultivo para la fase de enraizamiento de la variedad comercial de plátano FHIA 18. Ciget Pinar del $\begin{array}{llllll}\text { Rio, } & 10 & \text { (4): } 10 & \text { p. } & 2008 . & \text { Disponible }\end{array}$ http://www.ciget.pinar.cu/Revista/No.20084/art\%EDculos/utilizacion\%20de\%20aloe.pdf

16. Giraldo LA, Ríos HF, Polanco M. Efecto de dos enraizadores en tres especies forestales promisorias para la recuperación de suelos. Revista de Investigación Agraria y Ambiental, 1: 41-47. 2009.

17. Lema L. Evaluación de la eficacia de seis enraizadores y dos sustratos para la propagación de ramillas de café robusta (coffea canephora) en vivero, Cantón Francisco de Orellana, provincia de Orellana". Tesis Ingeniero Agrónomo, 87p. $2012 . \quad$ Disponible En: http://www.usfx.bo/nueva/vicerrectorado/citas/AGRARIAS 7/Ingenieria\%20Agr onomica/74.pdf 
18. Medina MG, García DE, Moratinos P, Cova LJ. Comparación de tres leguminosas arbóreas sembradas en un sustrato alcalino durante el período de aviveramiento. I. Variables morfoestructurales. Pastos y forrajes, 34 (1): $37-$ 52. 2011.

19. Medina MG, García DE, Clavero T, Iglesias JM, López JG. Evaluación inicial de la morera (Morus alba L.) en condiciones de vivero. Zootecnia Tropical, 25 (1): 43-49. 2007.

20. Medina MG, García DE, Clavero, Iglesias JM. Estudio comparativo de Moringa oleifera y Leucaena leucocephala durante la germinación y la etapa inicial de crecimiento. Zootecnia Tropical, 25 (2): 83-93. 2007.

21. Moreno F, Guerrero A. Evaluación de cuatro métodos de propagación en campo de Trichanthera gigantea. Rev. Fac. Agron., 22 (1): 13-22. 2005.

22. Navia JF, Restrepo JM, Villada DE, Ojeda PA. Agroforestería: Opción tecnológica para el manejo de suelos en zonas de laderas. Manual de Capacitación. Fundación para la Investigación y desarrollo Agrícola - FIDAR, Santiago de Cali, 78 p. 2003.

23. Organic SA. El vinagre como herbicida orgánico. 2008. Recuperado 20 de septiembre 2014. Disponible En: http://organicsa.net/el-vinagre-comoherbicida-organico.html

24. Rodríguez H, Hechevarria I. Gel de Aloe vera y harina de según como soporte sólido de medios de cultivo para plantas medicinales. Revista Cubana de Plantas Medicinales pág. 11 (1): 5 p. 2006.

25. Sabogal C, Guariguata MR, Broadhead J, Lescuyer G, Savilaakso S, Essoungou N, Sist P. Manejo forestal de uso múltiple en el trópico húmedo, oportunidades y desafíos para el manejo forestal sostenible. FAO Forestry Paper, 173. Roma, Italia. Organización de las Naciones Unidas para la Alimentación y la Agricultura / Bogor, Indonesia, Centro Internacional de Investigación Forestal. 2013.

26. Sánchez MD, Rosales M. Agroforestería para la producción animal en América Latina - II. Memorias de la segunda conferencia electrónica, Organización de las Naciones Unidas para la Agricultura (FAO), Roma. 2003.

27.Singh, U., Wilkens, P.W., Henao, J., Chien, S.H., Hellums, D.T., Hammond, L.L. An expert system for estimating agronomic effectiveness of freshly applied phosphate rock. In: S.S.S. Rajan y S.H. Chien, eds. Direct application of phosphate rock and related technology: latest developments and practical experiences. Proc. Int. Meeting, Kuala Lumpur, 16-20 July 2001. Muscle Shoals, Estados Unidos de América, IFDC. 441 p. 2003.

28. Taiz L, Zeiger E. Fisiología vegetal. Ed Universitat Jaume I, Castelló de la Plana, España. 583 p. 2006.

29. Toral OC. Comportamiento de especies arbóreas forrajeras en condiciones de vivero. Pastos y Forrajes, 32 (1): 1-11. 2009.

30. Uribe F., Zuluaga A., Murgueitio E., Valencia L., Zapata A., Solarte L., et al. Establecimiento y manejo de sistemas silvopastoriles. Manual 1. Proyecto ganadería colombiana sostenible. GEF, BANCO MUNDIAL, FEDEGAN, CIPAV, FONDO ACCION, TNC. Bogotá, Colombia. 78 p. 2011. 